\title{
AGP in daily clinical practice: a guide for use with the FreeStyle Libre flash glucose monitoring system
}

\author{
FRASER W GIBB, ${ }^{1}$ PETER JENNINGS, ${ }^{2}$ LALANTHA LEELARATHNA, ${ }^{3}$ EMMA G WILMOT ${ }^{4}$
}

\begin{abstract}
As real-time continuous glucose monitoring and flash glucose monitoring systems become more widely prescribed in the daily management of diabetes, it is important that the ambulatory glucose profile (AGP) methodology for reviewing and interpreting trends in glucose control is effectively applied. In this article we look at the essential features of the AGP and provide systematic and practical guidance on how the AGP can be interpreted in daily diabetes care with confidence. Using examples taken from glucose data captured by the FreeStyle Libre flash glucose monitoring system, we show how each aspect of the AGP can be used to understand daily patterns in glucose control for a person with diabetes, including the importance of time in range and adjunct use of individual daily logs. Using these elements collectively, we show how and why treatment adjustments can be made, with the goal of improving glycaemic control and diabetes outcomes.

Br J Diabetes 2020;20:32-40
\end{abstract}

Key words: ambulatory glucose profile, AGP, continuous glucose monitoring, flash glucose monitoring, CGM, glycaemic control, glycaemic variability, glycaemic stability, hypoglycaemia,

hyperglycaemia

\section{Background}

The ambulatory glucose profile (AGP) was first described in

Consultant Endocrinologist, Honorary Clinical Senior Lecturer, University of Edinburgh, Edinburgh Centre for Endocrinology and Diabetes, Edinburgh, UK

Diabetes Specialist Nurse, University Hospitals of Derby and Burton NHS Foundation Trust, Derby UK; Senior Lecturer, Nottingham Trent University, Nottingham, UK

3 Consultant Diabetologist and Honorary Senior Lecturer, Manchester Diabetes Centre, Manchester Royal Infirmary Science Centre, Manchester; Division of Diabetes, Endocrinology and Gastroenterology, Faculty of Biology, Medicine and Health, University of Manchester, Manchester, UK

4 Consultant Diabetologist, University Hospitals of Derby and Burton, Derby UK; Honorary (consultant) Assistant Professor, University of Nottingham, Nottingham, UK

Address for correspondence: Dr Emma G Wilmot Diabetes Department, Royal Derby Hospital, Uttoxeter Road, Derby DE22 3NE, UK

E-mail: emma.wilmot2@nhs.net

https://doi.org/10.15277/bjd.2020.240
1987, initially as a way of presenting validated self-monitored blood glucose data from meter downloads, ${ }^{1}$ but it has come into its own as a consequence of the evolution and widespread application of modern continuous glucose monitoring (CGM) and flash glucose monitoring technologies. Depending on the system used, CGM and flash glucose monitoring devices measure glucose levels in interstitial fluid in 1 or 5 minute increments on a continuous or on-demand basis over many days or weeks. These data can be used to describe details of an individual's glycaemic control, including their glucose exposure, glucose variability and stability.

The AGP is an internationally agreed standard for summarising and interpreting this wealth of glycaemic data in a visually impactful format that allows diabetes healthcare professionals and people with diabetes to identify patterns and trends in daily glucose control, including those that raise significant clinical concerns. ${ }^{2}$ In this way, the AGP can be used to target changes to daily diabetes therapy and to aspects of lifestyle that can improve overall glucose control and minimise the adverse consequences of dysglycaemia.

A number of protocols have been published that aim to optimise analysis of the AGP. ${ }^{3-5}$ In these, priority has been given to identifying the pattern and risk of hypoglycaemia, as well as the degree of variability underlying the glucose levels. However, such consensus recommendations do not always illustrate the principles of AGP using the most practical examples and clear terminology. The objective of this article is to provide a simple step-by-step guide that explains how to interpret an AGP in the context of a diabetes treatment review with a person living with diabetes and to illustrate these steps using clear examples. In doing so we will focus on how data are managed and represented by the FreeStyle Libre flash glucose monitoring system, which is an intermittently scanned CGM technology. It is important to reiterate that all of the principles covered in this review can also be applied to the sensor glucose data from real-time CGM systems that are currently available, and also viewed through different data management tools. However, in order to give these principles the most practical clinical utility, we have provided examples based on the visual tools provided by the FreeStyle Libre system.

\section{The essential features of an AGP}

Each AGP displays large amounts of glucose data from across several days or weeks as if all the readings had occurred in a single 24-hour period - the so-called 'modal' day. Using data collected on 257 individuals with type 1 diabetes in two studies, 
Figure 1. The key visual features of an ambulatory glucose profile (AGP) explained

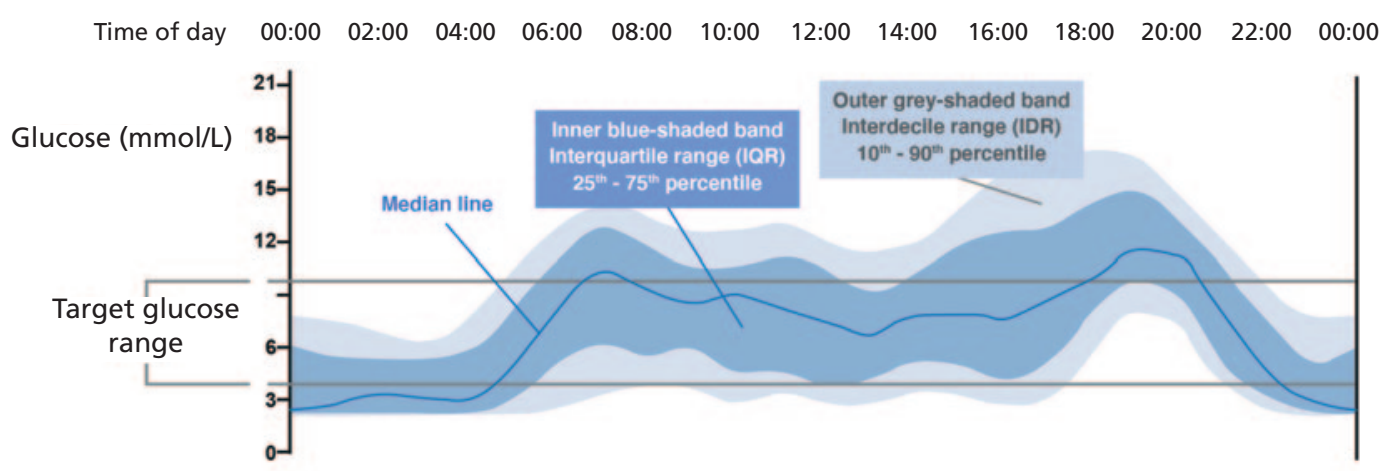

1. Blue line - this is the median line and reflects the average glucose at each point in the day. It provides a visual trace of whether average glucose is within the target range and how much it swings up and down throughout the day.

2. Inner blue-shaded band - this is the $25-75$ th percentile band, also called the interquartile range (IQR). It shows the $50 \%$ of all glucose readings levels that are closest to the median line and how variable they are from day-to-day. This blue IQR band shows the more consistent daily trends in glucose levels and indicates how medication and mealtimes are influencing glucose control. Because this is where glucose levels are 'half of the time', the thinner the blue-shaded band is, the less day-to-day variability is pictured.

3. Outside grey-shaded band - this is the 10-90th percentile, also called the interdecile range (IDR). The visible part of the IDR includes the readings that reflect less common departures from the daily average glucose. This is glucose variation that is happening on some days but not others and can indicate how behaviour and lifestyle issues are impacting on glucose control. The wider this grey band is, the more variable are these occasional readings.

4. Two parallel lines - the Target Glucose Range, where most of the glucose readings should be.

one using the FreeStyle Libre Pro system and one using the Dexcom G4 system, it has been established that 14 consecutive days of continuous glucose sensor data provides an optimal estimation of glucose control for interpretation in an AGP, as long as this includes a minimum of $70 \%$ of the sensor data from across this period. ${ }^{6}$

\section{What you see in an AGP}

Each AGP is a graphical and dynamic way of identifying times of greater risk of hypoglycaemia or glucose variability. The AGP comprises four key features: the median line; the interquartile range $(I Q R)$, typically shaded in dark blue; the interdecile range (IDR), typically shaded in light blue or grey; and the target glucose range. 1,2 These are described in Figure 1. It must be pointed out that the established AGP structure has used the IDR, as defined by the 10-90th percentiles, for the purpose of visualising less frequent glucose variability around the median line. However, there is a move to using the 5-95th percentiles to better identify infrequent, yet significant hypoglycemia, which may not be detected by the 10th percentile. ${ }^{7}$ Although most software for rendering AGP reports uses the IDR bounded by the 10-90th percentiles for analysis of less frequent glucose values, it is important to keep in mind the possibility that future updates may use the 5-95th percentiles for this purpose.

Each of the elements of the AGP can combine to tell a clear story about glucose control across each day and between different days. These defined features of the AGP can be used in a systematic and straightforward way to identify trends in glucose control. This includes not only the problem areas for remedial action, but also the parts of each day that reveal good control and highlight the selfmanagement choices that maintain glucose within target.

\section{Defining an AGP that is representative of typical glucose control}

Despite the potentially huge amount of glucose data collected by flash glucose monitoring and real-time CGM devices, it is important to realise that there is an optimum amount of information that should be contained in an AGP report.

\section{Sensor data capture}

The amount of data captured by the glucose sensor will dictate how accurately the AGP reflects real-world glucose control. Ideally this should be at least $70 \%$ across each day to generate an AGP report that enables optimal analysis and decisionmaking. ${ }^{6}$ In practice, $100 \%$ data capture is unrealistic, but many users can achieve a level of $90 \%$, which provides confidence in the AGP analysis. If there are any significant gaps in data at certain times of day, the AGP must be interpreted with caution at these time points. With flash glucose monitoring, glucose data are not transmitted continuously from the sensor and the user must actively scan the sensor with a reading device or smartphone app. Capture of complete 24-hour data can be achieved if the sensor is scanned once every 8 hours.

\section{4 consecutive days of data works best for an AGP review} It is unrealistic to expect that people with diabetes will have consistent glycaemic trends and patterns within each 24-hour period. As described above, 14 consecutive days of glucose data is optimal to provide a clinically relevant snapshot of glycaemic control for any individual using an AGP, although as little as 5 days of data is sufficient to generate a profile. ${ }^{6,8}$ However, it must be borne in mind that an AGP constructed from a small number of days will risk that any single day with a very distinct pattern may skew more general 
Figure 2. Glycaemic trends captured by ambulatory glucose profile (AGP) reporting over (a) 7, (b) 14 and (c) 28 consecutive days in the same individual within the same 28-day period. The AGP reports have been generated from data collected within the same consecutive 28-day period for a single individual. As the reporting period extends from 7 to 28 days, the visible trends in glucose variability change, with consequent implications for interpretation. 6,8

(a) 7 consecutive days: potential for single days with very distinct patterns to skew more general glycaemic trends

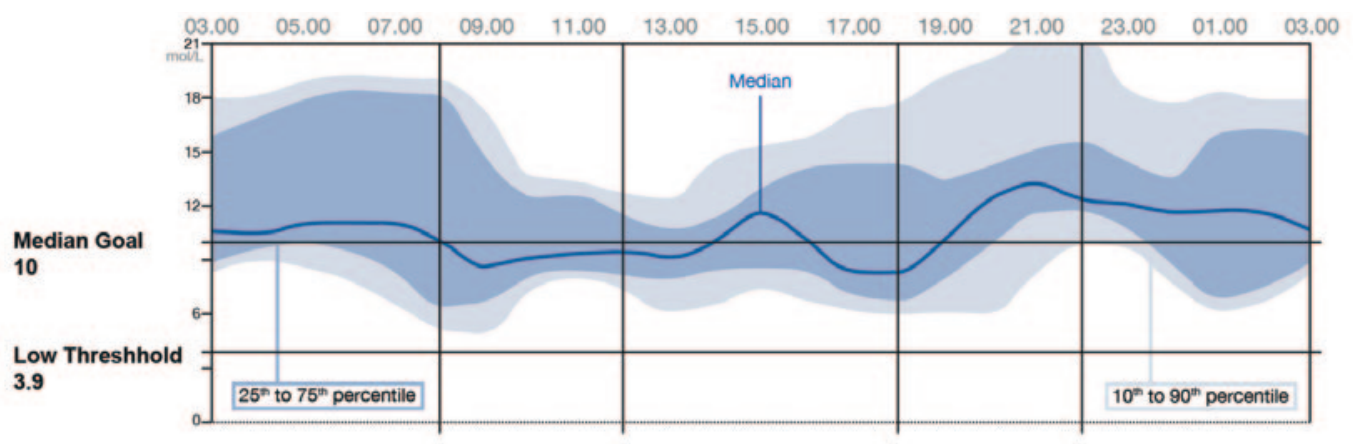

(b) 14 consecutive days: optimal to provide a clinically relevant snapshot of glycaemic control for any individual

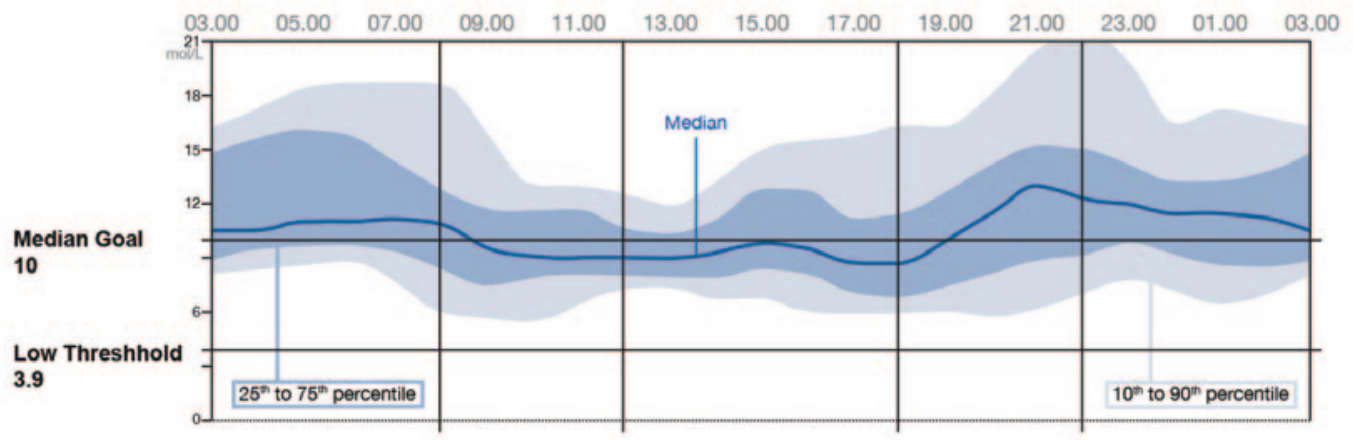

(c) 28 consecutive days: extended period can result in the dilution of meaningful trends

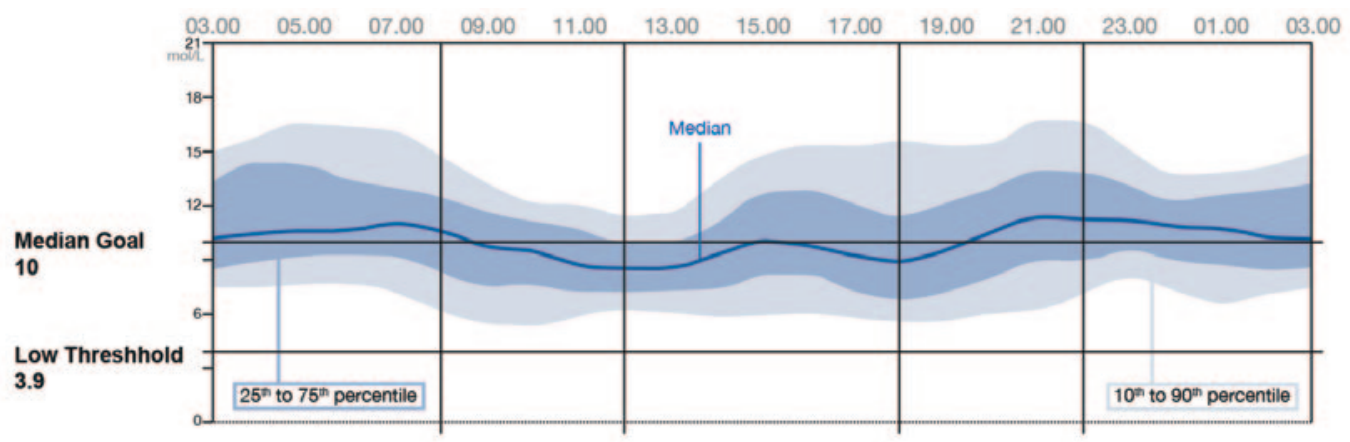

glycaemic trends and impair their interpretation. Similarly, an AGP that covers an extended period, say 28 days or more, can result in the dilution of meaningful trends, as illustrated in Figure 2. The sequence of AGP reports shows that, within a single individual, the shape of the AGP curves changes with the increasing availability of more data over 7, 14 and 28 days. With only 7 days of data (Figure 2a), the IQR and IDR bands are wider and ballooning above the median line, giving the impression of wider glucose variability. With 28 days of data (Figure 2c), the IQR and IDR bands are visibly narrower and more evenly distributed around the median line, suggesting less glucose variability. It is the AGP using 14 days of data
(Figure 2b) where the IQR and IDR bands have been shown to paint the most clinically relevant picture of glycaemic variability. ${ }^{6}$

Note also that the days selected for analysis in an AGP report should reflect the typical established therapeutic daily routine for any person with diabetes. Uncommon departure from routine - for example, holidays, days with different work schedules or periods of transition between different treatment plans - can influence the profile and should be avoided.

Overall, an AGP can be most informative when the most recent 14-day period for analysis that covers a period of routine diabetes management is selected. 


\section{Value of assessing time in range}

Time in range (TIR) refers to the percentage of time that a person with diabetes spends within their defined target glucose range (Figure 1) and is a good general indication of their overall glucose control. More importantly, two recent publications have shown that TIR is directly correlated with the risk of microvascular complications. A retrospective analysis of self-monitoring of blood glucose data from the Diabetes Control and Complications Trial (DCCT) study demonstrated a significant difference in TIR of 10-12\% between study subjects who developed complications compared with those who did not. The risk of progression of retinopathy was increased by $64 \%$ and the risk of developing microalbuminuria was increased by $40 \%$ for each $10 \%$ fall in TIR. ${ }^{9}$ Similarly, in a study using CGM to assess glucose control in 3,262 subjects with type 2 diabetes, TIR was shown to be inversely correlated with the prevalence and severity of diabetic retinopathy. ${ }^{10}$

Although there are default settings for the target glucose range, it is possible to set an individual target glucose range for each person using the FreeStyle Libre flash glucose monitoring system. However, international consensus recommendations have proposed that a target glucose range of $3.9-10 \mathrm{mmol} / \mathrm{L}$ is an appropriate standard against which to assess TIR, both in clinical practice and in clinical trials. ${ }^{11-13}$ More practically, the 2019 recommendations from the International Consensus on Time in Range ${ }^{14}$ have formalised the targets for TIR for people with type 1 diabetes or with type 2 diabetes, as well as for people at high risk of hypoglycaemia because of age, duration of diabetes, duration of insulin therapy or impaired awareness of hypoglycaemia (IAH). These are detailed in Table 1.

Although a given TIR can be associated with a range of haemoglobin $\mathrm{A} 1 \mathrm{c}\left(\mathrm{HbA}_{1 \mathrm{c}}\right)$ levels, for a person with diabetes who has a target glucose range of 3.9-10 mmol/L, a TIR of $70 \%$ will correspond to an $\mathrm{HbA}_{1 \mathrm{c}}$ of approximately $7.0 \%$ (53 $\left.\mathrm{mmol} / \mathrm{mol}\right)$, whereas a TIR of $50 \%$ will correspond to an $\mathrm{HbA}_{1 \text { c }}$ of $7.9 \%$ (63 $\mathrm{mmol} / \mathrm{mol}$ ). ${ }^{9}$ Consequently, \% TIR can be used with each individual as part of goal setting, as can improvements in \% time above or below target.

These TIR summary statistics are important to provide context and confidence when reviewing an AGP. It isn't always easy to compare different AGP visual charts from one review to the next, however the \%TIR and \% time below data can be directly compared, providing an objective perspective on glucose control between reviews. For users of the FreeStyle Libre system, these data are made available in the Snapshot Summary that accompanies the AGP report when using the LibreView platform for managing, sharing and reviewing collected glucose data (Figure 3). Users can also review their TIR on their handset or mobile phone, facilitating regular review of their progress and achievement of their personal goals.

\section{A step-by-step approach for reviewing and interpreting an AGP}

The AGP is a useful tool to highlight areas of concern for focus in a consultation. However, this interpretation must always be performed in the context of the user story and the glucose daily traces. Adopting a step-by-step approach can help clinicians and people with diabetes to systematically interpret the AGP.

\section{Step 1: Hypoglycaemia}

Identifying hypoglycaemia is the first step in any AGP review. Hypoglycaemia is the major limiting factor in the glycaemic management of type 1 and type 2 diabetes. ${ }^{15}$ Reducing both the occurrence of hypoglycaemia and the risk of hypoglycaemia is a central tenet of good diabetes care.

Is there any grey or blue in the hypoglycaemic zone below the target glucose range? If so, then there is a trend to low glucose at

Table 1 Consensus recommendations for time in range, time below range and time above range ${ }^{14}$

\begin{tabular}{|c|c|c|c|c|c|c|}
\hline \multirow[b]{2}{*}{ Diabetes group } & \multicolumn{2}{|c|}{ Time in range (TIR) } & \multicolumn{2}{|c|}{ Time below range (TBR) } & \multicolumn{2}{|c|}{ Time above range (TAR) } \\
\hline & Target range & $\begin{array}{l}\% \text { of readings: } \\
\text { time per day }\end{array}$ & $\begin{array}{l}\text { Below target } \\
\text { level }\end{array}$ & $\begin{array}{l}\% \text { of readings: } \\
\text { time per day }\end{array}$ & $\begin{array}{l}\text { Above target } \\
\text { level }\end{array}$ & $\begin{array}{l}\% \text { of readings: } \\
\text { time per day }\end{array}$ \\
\hline \multirow[t]{2}{*}{ Type 1/type 2} & $\begin{array}{l}3.9-10.0 \mathrm{mmol} / \mathrm{L} \\
(70-180 \mathrm{mg} / \mathrm{dL})\end{array}$ & $\begin{array}{l}>70 \%: \\
>16 \mathrm{~h}, 48 \mathrm{~min}\end{array}$ & $\begin{array}{l}<3.9 \mathrm{mmol} / \mathrm{L} \\
(70 \mathrm{mg} / \mathrm{dL})\end{array}$ & $\begin{array}{l}<4 \% \\
<1 h\end{array}$ & $\begin{array}{l}>10.0 \mathrm{mmol} / \mathrm{L} \\
(>180 \mathrm{mg} / \mathrm{dL})\end{array}$ & $\begin{array}{l}<25 \%: \\
<6 \mathrm{hr}\end{array}$ \\
\hline & & & $\begin{array}{l}<3.0 \mathrm{mmol} / \mathrm{L} \\
(54 \mathrm{mg} / \mathrm{dL})\end{array}$ & $\begin{array}{l}<1 \% \\
<15 \min \end{array}$ & $\begin{array}{l}>13.9 \mathrm{mmol} / \mathrm{L} \\
(>250 \mathrm{mg} / \mathrm{dL})\end{array}$ & $\begin{array}{l}<5 \% \\
<1 \mathrm{~h}, 12 \mathrm{~min}\end{array}$ \\
\hline $\begin{array}{l}\text { Older/high-risk } \\
\text { type } 1 \text { or type } 2 *\end{array}$ & $\begin{array}{l}3.9-10.0 \mathrm{mmol} / \mathrm{L} \\
(70-180 \mathrm{mg} / \mathrm{dL})\end{array}$ & $\begin{array}{l}>50 \% \\
>12 \mathrm{~h}\end{array}$ & $\begin{array}{l}<3.9 \mathrm{mmol} / \mathrm{L} \\
(70 \mathrm{mg} / \mathrm{dL})\end{array}$ & $\begin{array}{l}<1 \%: \\
<15 \min \end{array}$ & $\begin{array}{l}>13.9 \mathrm{mmol} / \mathrm{L} \\
(>250 \mathrm{mg} / \mathrm{dL})\end{array}$ & $\begin{array}{l}<10 \%: \\
<2 \text { h, } 24 \mathrm{~min}\end{array}$ \\
\hline \multirow[t]{2}{*}{ Pregnancy, type $1 \dagger$} & $\begin{array}{l}3.5-7.8 \mathrm{mmol} / \mathrm{L} \\
(63-140 \mathrm{mg} / \mathrm{dL})\end{array}$ & $\begin{array}{l}>70 \%: \\
>16 \mathrm{~h}, 48 \mathrm{~min}\end{array}$ & $\begin{array}{l}<3.5 \mathrm{mmol} / \mathrm{L} \\
(63 \mathrm{mg} / \mathrm{dL})\end{array}$ & $\begin{array}{l}<4 \% \\
<1 h\end{array}$ & $\begin{array}{l}>7.8 \mathrm{mmol} / \mathrm{L} \\
(>140 \mathrm{mg} / \mathrm{dL})\end{array}$ & $\begin{array}{l}<25 \%: \\
<6 \mathrm{~h}\end{array}$ \\
\hline & & & $\begin{array}{l}<3.0 \mathrm{mmol} / \mathrm{L} \\
(54 \mathrm{mg} / \mathrm{dL})\end{array}$ & $\begin{array}{l}<1 \% \\
<15 \min \end{array}$ & & \\
\hline
\end{tabular}

*People with type 1 or type 2 diabetes at high risk of hypoglycaemia because of age, duration of diabetes, duration of insulin therapy or impaired awareness of hypoglycaemia (IAH).

†\% TIR in pregnancy are based on limited evidence. No consensus recommendations for \% TIR in pregnancy in type 2 diabetes or in gestational diabetes are available. 
Figure 3. Glucose control snapshot for flash glucose monitoring data that accompanies an ambulatory glucose profile (AGP) report through LibreView. TBR, time below range; TIR, time in range

\section{Glucose - Estimated A1c 7.1\% or $54 \mathrm{mmol} / \mathrm{L}$}

$\begin{array}{ll}\text { Average glucose } & 8.7 \mathrm{mmol} / \mathrm{L} \\ \text { \% above target } & 42 \% \\ \% \text { in target } & 34 \% \\ \% \text { below target } & 24 \%\end{array}$

Low glucose events 27

Average duration $131 \mathrm{~min}$.
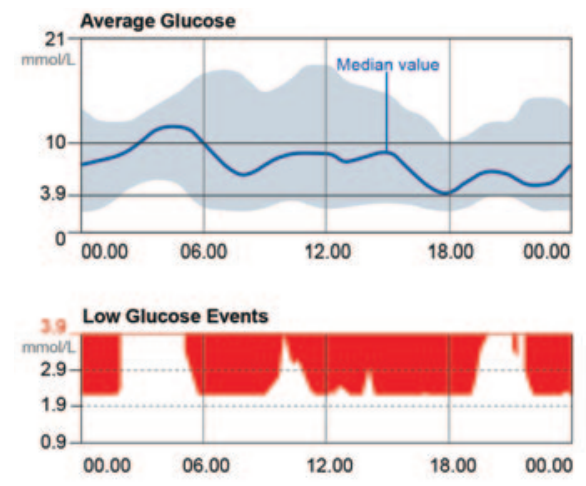

\section{Sensor usage}

Sensor data captured $79 \%$

Daily scans

9
Hypoglycaemia - TBR $>4 \%$ or $>1 \%$ if high risk - need to identify and tackle causes of hypoglycaemia, as directed by the user story.

Hyperglycaemia - TIR is low but $\mathrm{HbA}_{1 \mathrm{c}}$ is reasonable, highlighting the disconnect between $\mathrm{HbA}_{1 \mathrm{c}}$ and optimal glucose control. To increase TIR we need to reduce hypoglycaemia.

Variability - explore causes of high daytime variability. Check injection sites, bolus timing, ratios. What does the user think is causing the day-to-day variability? Look at daily traces for more information and to guide discussion. these times. How predictable are these hypoglycaemic trends? A low median line with blue and grey bands extending below the low-glucose threshold indicates a consistent trend. However, there is only limited space on the AGP for low-glucose readings to be captured and distributed below $3.9 \mathrm{mmol} / \mathrm{L}$, so the actual width of the blue and grey bands is less revealing. Therefore, trends to low glucose in the AGP should be interpreted in the context of time below range. The international consensus recommends aiming for $<4 \%$ time below range generally and $<1 \%$ in high-risk individuals. The AGP can help identify time points in the day when hypoglycaemia is problematic. Consider dose and timing of basal insulin (lows overnight and early morning) or mealtime insulin (lows after or between meals). If the grey band alone is in the hypo zone, then additionally investigate factors associated with lifestyle or behaviour (eg, work, exercise, over-correction of high glucose) which can be reviewed in more detail in the daily glucose traces.

Any trends to low glucose below $3.9 \mathrm{mmol} / \mathrm{L}$ can be further investigated by looking at the low glucose events log that is provided in the snapshot report that accompanies the AGP when accessed using the LibreView tool for sharing and reviewing glucose data collected by the FreeStyle Libre system. This also provides an objective readout on the typical time, depth and duration of hypoglycaemic episodes, and whether glucose has fallen below the clinically significant $3.0 \mathrm{mmol} / \mathrm{L}$ level. One noteworthy observation is that overnight glucose data can occasionally be incomplete. These gaps can result from scan intervals longer than 8 hours, with consequent loss of data. Another user-reported phenomenon is episodic loss of glucose sensitivity overnight if they sleep on their sensor. This is believed to result from compression of the sensorapplication site, with reduced interstitial fluid volume. Given the importance of understanding nocturnal patterns of low glucose, it is worth highlighting these issues with any subject experiencing overnight hypoglycaemia. It is worth pointing out that the accuracy of CGM and flash glucose monitoring sensors is subject to larger errors as glucose falls towards the hypoglycaemic range. ${ }^{16}$ Concurrence data on FreeStyle Libre sensors indicate that this can result in a low glucose reading when capillary blood glucose is still above $3.9 \mathrm{mmol} / \mathrm{L} .{ }^{17}$ Consequently, confirmation of hypoglycaemia with a fingerprick blood glucose test is always recommended by manufacturers.

\section{Step 2: Hyperglycaemia}

Identifying hyperglycaemia is the second step in any AGP review. There is no doubt that long-term exposure to high glucose has adverse consequences. Both the DCCT and the UKPDS allow us to conclude that reducing hyperglycaemia, as measured by $\mathrm{HbA}_{1 \mathrm{c}}$, is associated with clinically significant reductions in microvascular complications and long-term macrovascular disease. ${ }^{18-20}$ Similarly, as stated previously, improving time in range will reduce the risk of adverse microvascular outcomes. ${ }^{9,10}$ In the AGP, look for trends towards high glucose above the upper limit of the target glucose range of $10 \mathrm{mmol} / \mathrm{L}$, especially if they are persistent as indicated by a high average glucose line bordered by a narrow blue band (see the first example in Table 2). These will be caused by issues with the treatment parameters. If high glucose is shown by the grey band, it indicates less common exposure caused by lifestyle and behavioural activities.

\section{Step 3: Glucose variability}

Glycaemic variability is emerging as an important risk factor for cardiovascular complications of diabetes, independent of longterm $\mathrm{HbA}_{1 \mathrm{c}}$ or sustained hyperglycaemia. ${ }^{21}$ A low $\mathrm{HbA}_{1 \mathrm{c}}$ can 
Table 2 Examples of descriptive analogies that can make sense in an ambulatory glucose profile (AGP) review in preference to technical language. IQR, interquartile range; IDR, interdecile range

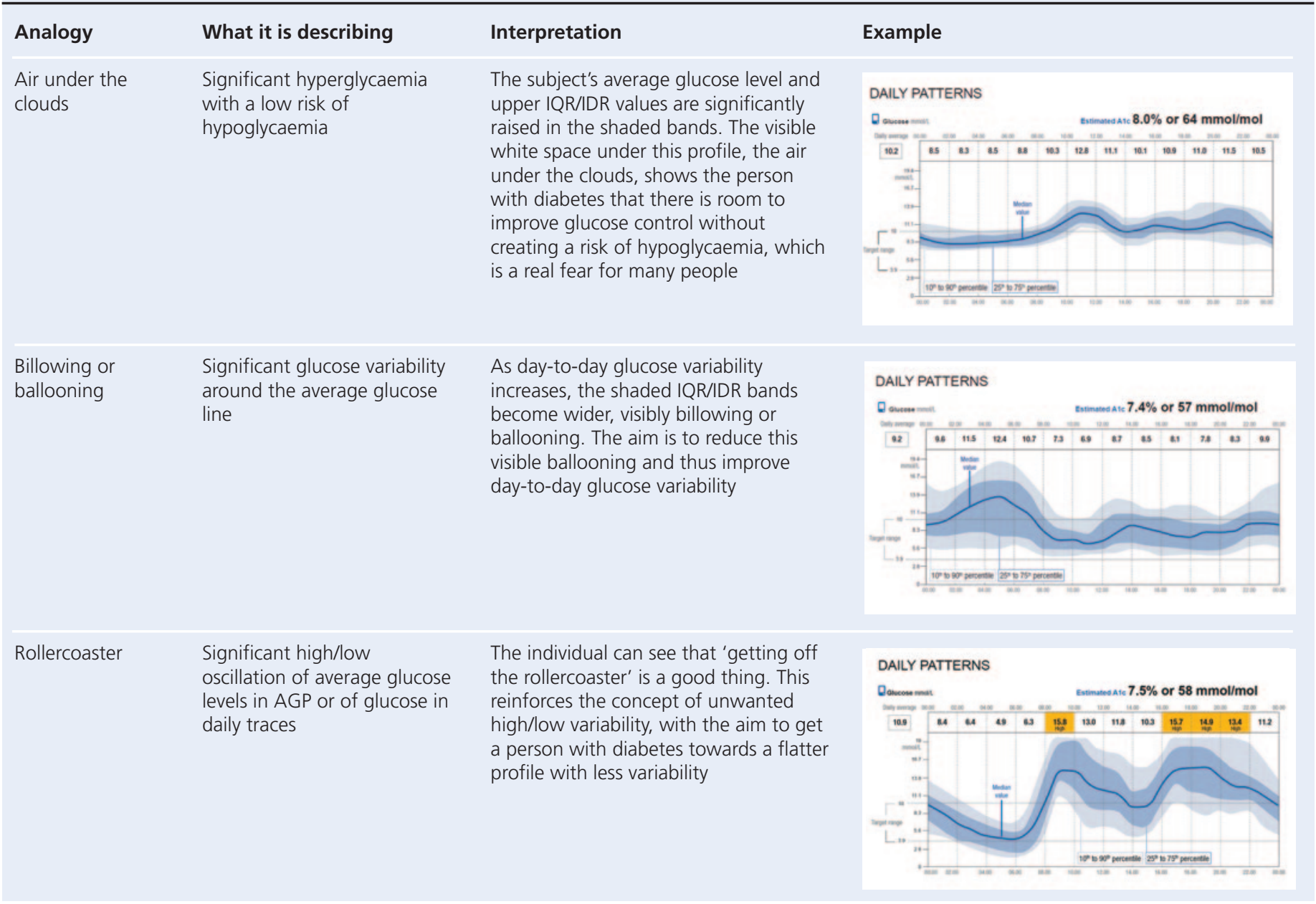

mask significant swings in glucose and give no indication of this associated cardiovascular risk. If the blue average glucose line is on a 'rollercoaster', there are unwanted high/low swings in glucose consistently at these times. Mealtime excursions indicate a need to change pre-meal insulin timings and/or doses. Peaks after hypoglycaemia may indicate a need to review management of low glucose to avoid overtreatment.

The width of the blue and grey bands in an AGP is an indication of the amount of day-to-day glucose variability as well as less common variability. The thinner the blue and grey bands are, the better. A wider 'billowing' or 'ballooning' blue band means unwanted glucose fluctuations on most days, which suggests a need to adjust management of therapeutic parameters such as insulin doses and/or timings, or review meal planning. Wider grey bands represent occasional factors such as unplanned meals and snacks, intermittent exercise or weekday routines versus weekends. When investigating the causes of variability indicated by a wide blue or grey band, a more comprehensive assessment can be achieved by reviewing the daily logs for the period in question. These can reveal the detail behind the pattern seen in the AGP. The possible scope and interpretation of glucose variability that may be seen within any AGP is outlined in Figure 4, along with the different causes that should be investigated.

\section{Digging deeper into the details - the essential role of the daily logs}

Once the AGP has identified potential issues, the causes can be investigated by looking at the daily log of glucose readings. These individual traces add important detail to the insights from the AGP and can be used to further understand each individual story. For example, significant variability can be a facet of weekday versus weekend activities (Figure 5). These are not revealed through the modal day of the AGP, so it can make sense to investigate these at the level of daily logs. Similarly, a pattern of low glucose on the AGP may reflect the combined impact of insulin dosing, exercise and carbohydrate intake that can be confirmed by looking at the daily logs that correspond to the exercise schedule. Daily logs are most valuable when the user has taken time to enter information about their insulin dosing and times, as well as carbohydrate intake and exercise. Unfortunately, this is rare in real-life but there is still value in these day-by-day glucose traces. 
Figure 4. Possible scope and interpretation of glucose variability within an ambulatory glucose profile (AGP). $I Q R$, interquartile range; IDR, interdecile range

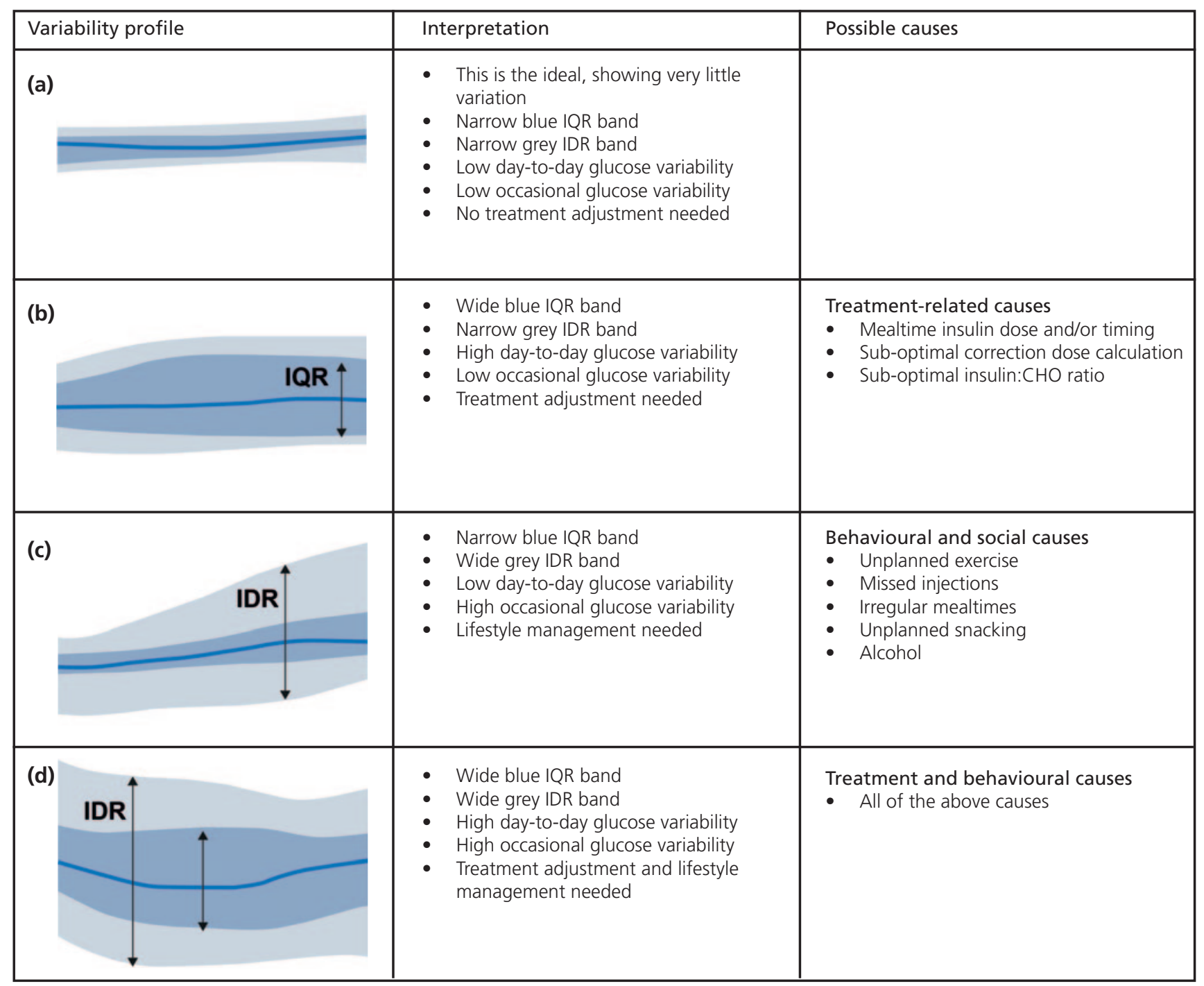

\section{Getting the most from a FreeStyle Libre data review}

Time with the person with diabetes is always limited, so it is vital to make the most of this precious time.

- Start with the individual and always sense-check your assumptions: Let the person with diabetes voice their concerns and help them to interpret these in the context of the AGP as much as possible, since their agenda is paramount., Only they can provide the personal insights that give meaning to the patterns and trends revealed in their own AGP and daily traces. This will empower them to take control of day-to-day decisions in their diabetes self-management.

- Perspective: It is important to keep perspective on the results during an AGP review and look back at AGPs from previous appointments to review progress made. With the FreeStyle Libre system, the easiest way to manage this is to use the LibreView platform for storing and analysing flash glucose monitoring data.
Also, users may not look at their AGP outside of the clinic review, but always remind them to reflect on how their lifestyle can influence the AGP.

- Focus on glucose levels overnight: The period between going to bed and waking before breakfast covers around a third of the day. If this part of the day is under control, then you can focus on the task of dealing with everything that impacts on daytime glucose control. A consistent trend to low glucose overnight can indicate a need to reduce basal insulin, whereas overnight high glucose is often the result of an inadequate bolus insulin dose with the evening meal or pre-bed snacking without adequate cover. Again, these overnight trends in the AGP should always be interpreted in the context of the daily traces.

- Think medication, lifestyle and behaviour: When you see high or low glucose, use the shaded bands to guide you to the cause. A narrow blue band indicates a trend that is happening 
Figure 5. Daily logs showing how different days impact on glucose control*

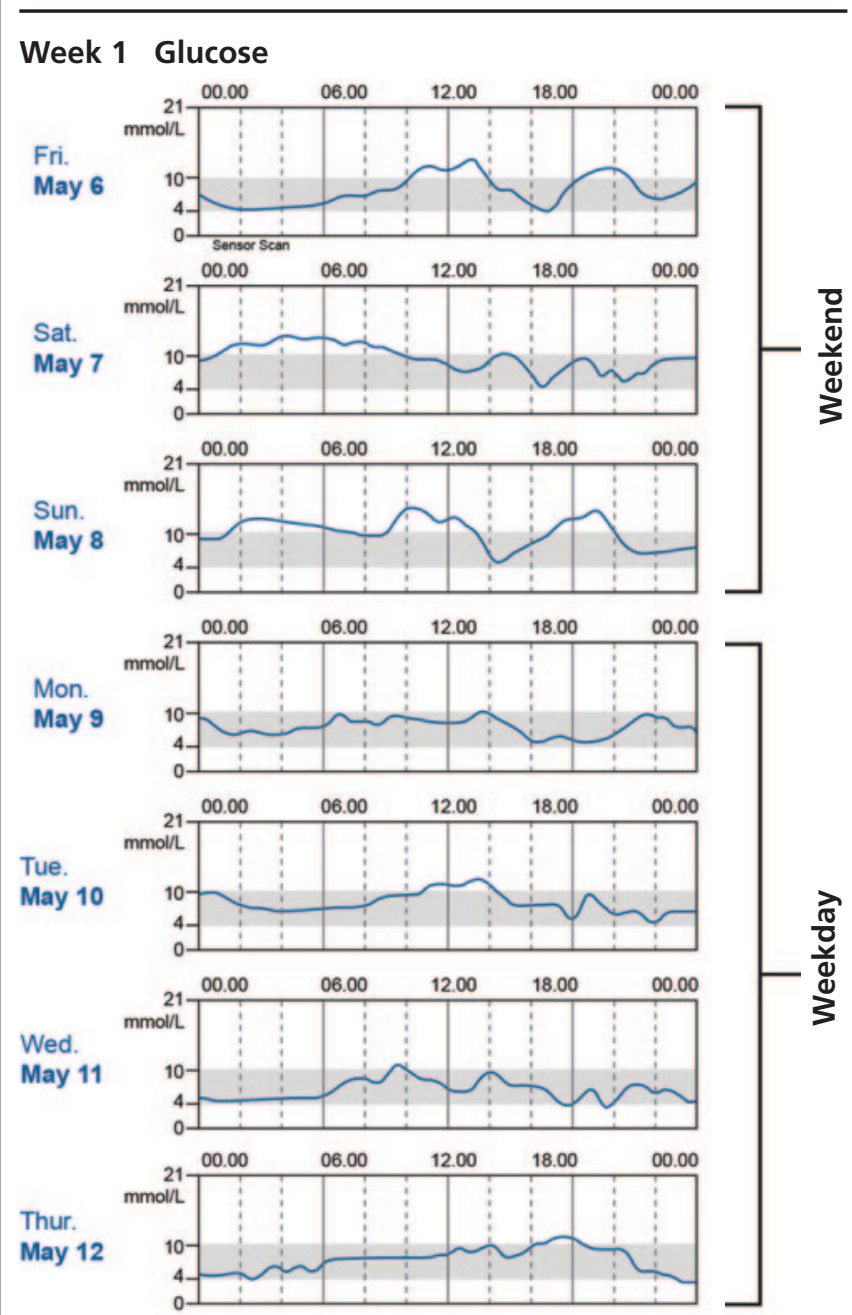

In this example, the daily logs show that the weekend glucose profiles for Friday, Saturday and Sunday differ from the profiles visible through the working week.

*Daily logs are accessible using the LibreView data management platform for data collected using the FreeStyle Libre system.

consistently each day, so consider medication and mealtimes. Conversely, a wider blue or outer grey band reflects glucose variation for distinct reasons on different days, so focus on aspects of behaviour or lifestyle such as a missed insulin injection, periodic exercise, social events or illness. In discussion with the individual, the daily logs can provide insights into possible times and activities that have contributed to the variability shown in the AGP.

- User-friendly analogies: To make the most of the consultation, it is critical to dispense with the technical jargon that is attached to the features of an AGP and bring it to life with easy-to-understand language and descriptions. In an AGP review, use analogies for what you are discussing that will be understood see, for example, those in Table 2.

\section{Key messages}

- The ambulatory glucose profile, combined with \% time in range are invaluable tools which support decision making and optimisation of glycaemia

- The international consensus on time in range recommends aiming for $>70 \%$ time in range (3.9-10 mmol/l) with $<4 \%$ time below range $(<3.9 \mathrm{mmol} / \mathrm{l})$; in high risk individuals target time in range is $>50 \%$ with $<1 \%$ time below range

- A step wise approach to glucose data includes the assessment of hypoglycaemia risk, then hyperglycaemia followed by glycaemic variability. Analysis of the daily traces can provide additional detail to support therapeutic decisions

\section{Deciding on which issues to tackle}

During a diabetes review it makes sense to propose changes to only one aspect of glucose control at a time and assess the outcome at an appropriate time. This can either be at a scheduled in-clinic review or, if the FreeStyle Libre user is part of a LibreView practice, in a remote consultation in which the AGP can be shared with them. Some factors, such as changes in activity or behaviour, may need longer to assess before their impact can be measured.

The step-by-step approach to AGP detailed in this guide emphasises the importance of hypoglycaemia as a target for control. Episodes of hypoglycaemia, often followed by rebound hyperglycaemia as a consequence of overtreatment, can be unpredictable and complicate the task of glucose management for people with diabetes and their healthcare professionals. Flash glucose monitoring and real-time CGM are technologies that can reduce the frequency of hypoglycaemia in day-to-day glucose management. As part of this benefit, the visual format of the AGP allows the eye to quickly focus on continuing areas of concern that can be assessed in terms of how significant they are by their timing, duration and how low glucose has fallen.

Making judgements about targeting adverse hyperglycaemia and glucose variability is also a part of an effective AGP review. Better management of these aspects of glucose control can also have a direct effect on the Time in Range performance of each person with diabetes.

\section{Conclusion}

AGP has the potential to be a very effective way to visualise and interpret important daily trends in glucose control and glucose variability. For optimal analysis and effective decision making, each AGP should be based on at least $70 \%$ of sensor data capture over the period under review, ideally 14 consecutive days of routine daily diabetes management. The four key features of an AGP are: the median line; the interquartile range (IQR); the interdecile range (IDR); and the target glucose range. Each of these 
can be used in a systematic and straightforward step-by-step process to identify trends in glucose control. Where necessary, associated elements of the snapshot of glucose control, as well as the individual daily logs that are provided by the data management software, can be used to further investigate the detail behind the patterns and trends in the AGP.

Conflict of interest: FWG: consultancy fees from Dexcom. PJ: personal fees from Abbott Diabetes Care outside the submitted work. LL: speaker honoraria from Minimed Medtronic, Animas, Roche, Sanofi, Insulet and Novo Nordisk; advisory panel activities for Abbott Diabetes Care, Roche, Sanofi, Minimed Medtronic, Animas and Novo Nordisk; grants to attend educational meetings from Sanofi, Novo Nordisk and Takeda. EGW: speaker honoraria from Abbott Diabetes Care, Diasend, Dexcom, Eli Lilly, Minimed Medtronic, Novo Nordisk, Sanofi Aventis; has served on advisory panels for Abbott Diabetes Care, Eli Lilly, Medtronic and Sanofi Aventis; received grants to attend educational meetings from Boehringer Ingelheim, Diasend, Novo Nordisk, Roche and Sanofi Aventis.

Funding: None.

Acknowledgments: Editorial assistance with the writing of the manuscript was provided by Dr Robert Brines, Medical Director for Bite Medical Consulting. This support was paid for by Abbott diabetes care division, who had no input into the content of the manuscript.

\section{References}

1. Mazze RS, Lucido D, Langer O, Hartmann K, Rodbard D. Ambulatory glucose profile: representation of verified self-monitored blood glucose data. Diabetes Care 1987;10:111-17.

https://dx.doi.org/10.2337/diacare.10.1.111

2. Bergenstal RM, Ahmann AJ, Bailey T, et al. Recommendations for standardizing glucose reporting and analysis to optimize clinical decision making in diabetes: the Ambulatory Glucose Profile. Diabetes Technol Ther 2013;15:198-211. https://dx.doi.org/10.1089/dia.2013.0051

3. Mazze RS, Strock E, Wesley D, et al. Characterizing glucose exposure for individuals with normal glucose tolerance using continuous glucose monitoring and ambulatory glucose profile analysis. Diabetes Technol Ther 2008;10:149-59. https://dx.doi.org/10.1089/dia.2007.0293

4. Matthaei S, DeAlaiz R, Bosi E, Evans M, Geelhoed-Duijvestijn N, Joubert $M$. Consensus recommendations for the use of ambulatory glucose profile in clinical practice. Br J Diabetes 2014;14:153-7.

https://dx.doi.org/10.15277/bjdvd.2014.046

5. Evans M, Cranston I, Bailey CJ. Ambulatory glucose profile (AGP): utility in UK clinical practice. Br J Diabetes 2017;17:26-33. https://dx.doi.org/10.15277/bjd.2017.121

6. Riddlesworth TD, Beck RW, Gal RL, et al. Optimal sampling duration for continuous glucose monitoring to determine long-term glycemic control. Diabetes Technol Ther 2018;4:314-16.

https://dx.doi.org/10.1089/dia.2017.0455

7. Johnson ML, Martens TW, Criego AB, Carlson AE, Simonson GD, Bergen- stal RM. Utilizing the ambulatory glucose profile to standardize and implement continuous glucose monitoring in clinical practice. Diabetes Technol Ther 2019;21(Suppl 2):S217-25.

https://dx.doi.org/10.1089/dia.2019.0034

8. Dunn TC, Crouther N. Assessment of the variance of the ambulatory glucose profile over 3 to 20 days of continuous glucose monitoring. 46th EASD Conference, Stockholm. Poster 1054.

9. Beck RW, Bergenstal RM, Cheng P, et al. The relationships between time in range, hyperglycemia metrics, and HbA1c. J Diabetes Sci Technol 2019;13:614-26. https://dx. doi.org/10.1177/1932296818822496

10. Lu J, Ma X, Zhou J, et al. Association of time in range, as assessed by continuous glucose monitoring, with diabetic retinopathy in type 2 diabetes. Diabetes Care 2018;41:2370-6.

https://dx.doi.org/10.2337/dc18-1131

11. Danne $T$, Nimri $R$, Battelino $T$, et al. International consensus on use of continuous glucose monitoring. Diabetes Care 2017;40:1631-40. https://dx.doi.org/10.2337/dc17-1600

12. Petrie JR, Peters AL, Bergenstal RM, et al. Improving the clinical value and utility of CGM systems: issues and recommendations: a Joint Statement of the European Association for the Study of Diabetes and the American Diabetes Association Diabetes Technology Working Group. Diabetes Care 2017;40:1614-21. https://dx.doi.org/10.2337/dci17-0043

13. Agiostratidou $G$, Anhalt $H$, Ball $D$, et al. Standardizing clinically meaningful outcome measures beyond $\mathrm{HbA} 1 \mathrm{c}$ for type 1 diabetes: a Consensus Report of the American Association of Clinical Endocrinologists, the American Association of Diabetes Educators, the American Diabetes Association, the Endocrine Society, JDRF International, The Leona M. and Harry B. Helmsley Charitable Trust, the Pediatric Endocrine Society, and the T1D Exchange. Diabetes Care 2017;40:1622-30. https://dx.doi.org/10.2337/dc17-1624

14. Battelino T, Danne T, Amiel SA, et al. Clinical targets for continuous glucose monitoring data interpretation: recommendations from the International Consensus on Time in Range. Diabetes Care 2019; 42:1593-603. https://dx.doi.org/10.2337/dci19-0028

15. American Diabetes Association. Glycemic targets: standards of medical care in diabetes-2019. Diabetes Care 2019;42:561-70. https://dx.doi.org/10.2337/dc19-S006

16. Rodbard D. Characterizing accuracy and precision of glucose sensors and meters. J Diabetes Sci Technol 2014;8:980-5. https://doi/org/10.1177/1932296814541810

17. FreeStyle Libre 14 Day Flash Glucose Monitoring System user's manual DOC39764 Rev. B 07/18.

18. Diabetes Control and Complications Trial Research Group, Nathan DM, Genuth S, Lachin J, et al. The effect of intensive treatment of diabetes on the development and progression of long-term complications in insulin-dependent diabetes mellitus. N Engl J Med 1993;329:977-86. http://dx.doi.org/10.1056/NEJM199309303291401

19. Stratton IM, Adler Al, Neil AH, et al. Association of glycaemia with macrovascular and microvascular complications of type 2 diabetes (UKPDS 35): prospective observational study. BMJ 2000;321:405-12. https://dx.doi.org/10.1136/bmj.321.7258.405

20. Holman RR, Paul SK, Bethel AM, Matthews DR, Neil AW. 10-Year followup of intensive glucose control in type 2 diabetes. N Engl J Med 2008;359:1577-89. https://dx.doi.org/10.1056/NEJMoa0806470

21. Ceriello A, Monnier L, Owens D. Glycaemic variability in diabetes: clinical and therapeutic implications. Lancet Diabetes Endocrinol 2018;7:22130. https://dx.doi.org/10.1016/S2213-8587(18)30136-0 